\title{
THE HIPPARCOS DOUBLE AND MULTIPLE STAR SOLUTIONS
}

\author{
F. MIGNARD \\ $O C A / C E R G A$ \\ UMR CNRS 6527 \\ Av. Copernic, 06130-Grasse (France)
}

\begin{abstract}
The Hipparcos Catalogue provides general astrometric and photometric information on double and multiple stars in specific fields of the main Catalogue and detailed data on the components in the various sections of a dedicated annex : the Double and Multiple Systems Annex (DMSA). Overall statistics of these solutions are presented for the 13211 entries of this annex and the different types of solutions are outlined.
\end{abstract}

\section{THE HIPPARCOS DOUBLE STARS}

It was known well before the Hipparcos mission that a significant fraction of the observing programme will have to deal with double and multiple systems, a circumstance that seriously complicated the data treatment. When a Hipparcos target was a single object, the standard astrometric model in the data reduction assumed a uniform rectilinear motion in space relative to the barycentre of the solar system. In this case only five parameters were necessary to describe the full astrometric information embedded in the Hipparcos measurements : the two angular coordinates to specify the coordinate direction at the Catalogue epoch $\left(T_{0}=\mathrm{J} 1991.25\right)$, the two components of the proper motion and the parallax. In the case of a non single object the situation was more complex and additional parameters were required first to recognize that the target comprised at least two objects and to model properly the grid observations in order to achieve a good decoupling between the absolute and relative astrometry and photometry. The principles applied to the detection and the astrometry of the double stars have been presented in detail in the literature (Mignard et al, 1992, Söderhjelm et al., 1992 and Mignard et al, 1995) and the most updated information is now available in the Hipparcos documentation (ESA, 1997) and for the classification of the double star solutions in Lindegren et al (1997).

TABLE 1. Content in entries of the different parts of the Hipparcos Catalogue.

\begin{tabular}{clrc}
\hline Catalogue & Solutions & Entries & Comments \\
\hline Main Catalogue & Single star solutions & 100038 & 6763 suspected non single \\
DMSA : & & & \\
C & Component solutions & 13211 & 2996 new systems \\
G & Acceleration solutions & 2622 & Likely astrometric binaries \\
O & Orbital solutions & 235 & \\
V & Variable movers & 288 & \\
X & Stochastic solutions & 1561 & \\
\hline
\end{tabular}

About 15 percent of the Hipparcos Catalogue refers to objects seen non-single by the satellite, although in the remaining 85 percent there are likely many double and multiple systems not detectable and treated as single stars. Because of the variety of non-single stars, several types of solutions had to be devised to cope with this problem according to the separation, magnitude difference, orbital period. The resolved systems for which both the separation and magnitude difference 
were measured constitute the largest single group and the solutions are given in term of absolute astrometry of the components in the part C of the DMSA. Most systerns (12005) are double stars and 190 systems were solved for three or four components, corresponding in total to the 13211 entries of the Catalogue. Most of these systems were solved as fixed components (separation and position angle fixed over the mission) while 1186 include a linear relative motion of the components and for the remaining 202 the two parallaxes were solved independently of each other.

Other non single star solutions are referred to as acceleration solution when the absolute motion on the sky was significantly non-linear, orbital solutions when one or several orbital elements of the photocenter could be fitted to the data, variability-induced movers for which the photocenter exhibited a systematic motion due to the variability of at least one component and the stochastic solutions when no acceptable single or double star solutions were found. The respective content and denomination of each part are given in Table 1. Further ground-based observations should provide a better on the still uncertain status (single or multiple) of most of the entries in the part $\mathrm{G}, \mathrm{V}$ and $\mathrm{X}$ of the annex. In addition a special effort should be devoted to the subset of suspected non-singles, in particular with speckle interferometry.

The Double and Multiple star solutions are published in the printed form in various parts of the 17-volume Hipparcos Catalogue. The ASCII files are also available on the set of CD-ROMs published simultaneously, which also includes the intermediate data (abscissae and grid signals for 30 percent of the stars) that could be used for a reprocessing using a different modelling or to take advantage of new ground based data useful to constraint the solution.

\section{THE ASTROMETRIC SOLUTION}

Among the $\sim 11100$ entries of the main Catalogue solved for two components, there are $\sim 8100$ which were known as non single stars from ground based observations carried out before or during the Hipparcos mission and about 3000 new systems recognized as double from the Hipparcos data. The plots in Figure 1 show the distribution of these systems on a diagram magnitude difference - separation. They reveal a striking difference between the two populations : rather larger separations for the known systems, comprising mainly visual detections, with the exception of the speckle measurements in the left part of the diagram. The Hipparcos newly detected systems, on the contrary are mainly close visual binaries with separation $<0.5$ arcsec. The envelope of the distribution follows closely the detection limit of the Hipparcos optical and grid system. This indicates also the level of completeness to be considered in further statistical studies.
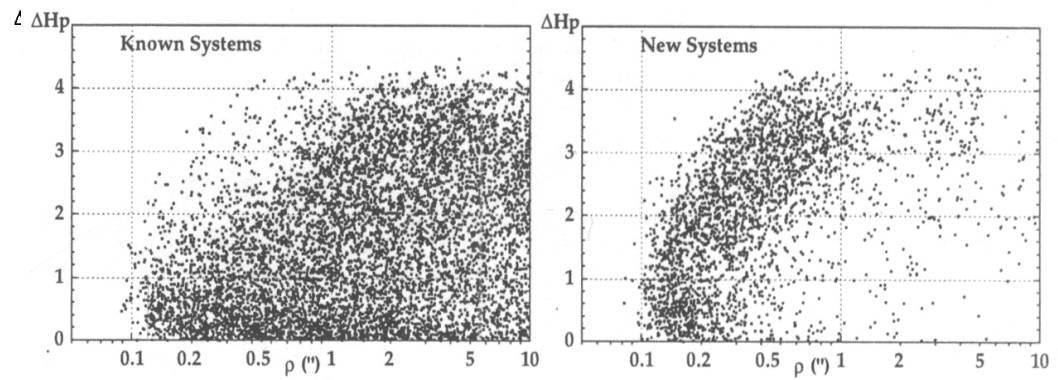

Figure 1. Distribution of the magnitude difference versus separation for the components of the double stars measured by Hipparcos. The left panel refers to systems known from ground based measurements and the right one to the systems discovered by Hipparcos.

The astrometric solutions have been derived by correcting the single star for the effect of the companion allowing to produce the absolute astrometry of either the primary or the photocenter, according to the separation. For the users, the part $\mathrm{C}$ of the annex gives the astrometric solution of each components derived from the merging of the FAST and NDAC solution after the two groups agreed on a list of indisputable detected non single stars. A slight possibility of grid step errors remains for few stars, which affect both the separation and the absolute position. For catalogue entries resolved in strictly two components, the relative astrometry and photometry is also given in the main Catalogue and is perfectly consistent with the data of the Annex. 
Apart for the relative astrometry, the parallax is the most relevant parameter for the double and multiple systems because of its implication in stellar physics. Because of the complication in the modelling of the double stars, it was reasonable to expect a significant degradation in the quality of the parallax compared to that of the single stars. The plots in Figure 2 compare the relative error in the parallax $\sigma_{\pi} / \pi$ for the two categories. All the entries $(=96731)$ not included in the Double and Multiple Systems Annex and with a non-negative parallax make the set of single stars, whereas the set multiple stars comprises the entries $(=12569)$ of the DMSA-C with non negative parallax. The difference is noticeable but not very large, given the difference in the modelling. There are 18 per cent of the single stars with $\sigma_{\pi} / \pi<0.1$ versus 13 per cent for the multiple and the level of 18 per cent of the multiples is reached at $\sigma_{\pi} / \pi<0.125$ instead of 0.10 for the singles. This results from a very fortunate circumstance which made the parallax solution moderately sensitive to the quality of the double star solution, unlike the other astrometric parameters. Thus, one can say, that despite a small difference of quality, the Hipparcos parallaxes are statistically homogeneous irrespective to whether the star was processed as single or not.
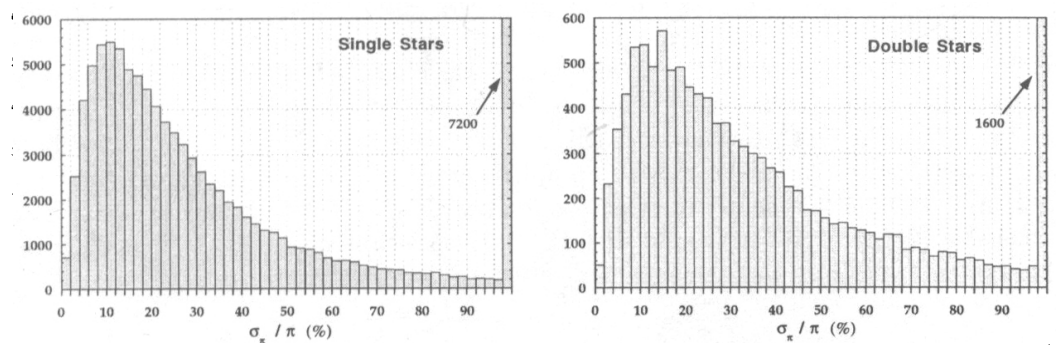

Figure 2. Relative standard errors of the Hipparcos parallaxes for the single stars and the double and multiple systems of the Annex-C.

Regarding the relative astrometry of double stars, the separations solved with Hipparcos range from 0.1 to 20 arcsec, with a median of 0.97 arcsec. The distribution of the standard errors in $\rho$ is shown in Fig. 3 for all the separations of the known double stars (left) and the new systems detected by Hipparcos (right). The difference between the two sets is much apparent and linked to the fact that the new double stars have predominantly small separations (then more difficult to resolve) with a median of 0.33 arcsec versus 1.55 arcsec for the complementary set. The medians of the standard errors are respectively 23 and 8 mas.
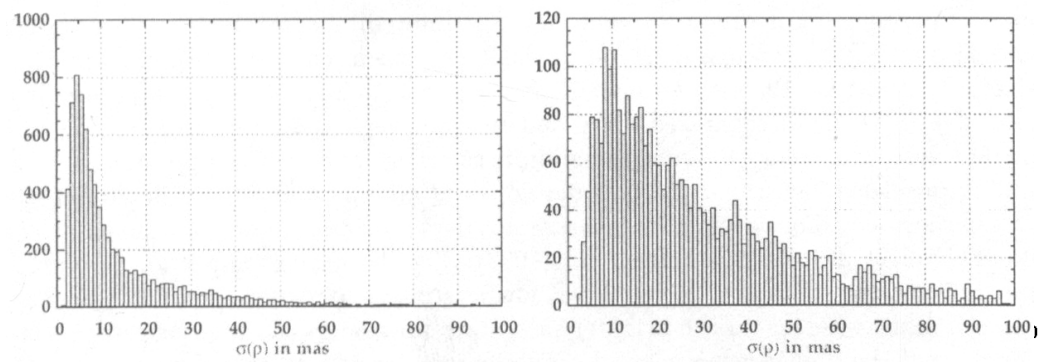

Figure 3. Standard errors in the separations of the double systems solved in the Hipparcos Catalogue respectively for known (left) and new systems (right).

\section{References}

ESA, 1997, The Hipparcos and Tycho Catalogues, SP-1200, Vols. 1-4

Lindegren L., Mignard F., Söderhjelm S. et al., 1997, A\&A, 323, L53.

Mignard, F., Froeschlé, M., Badiali, M. et al, 1992, A\&A, 258, 165.

Mignard, F., Söderhjelm, S., Bernstein, H. et al, 1995, A\&A, 304, 94.

Söderhjelm S., Evans, D.W., van Leeuwen F. and Lindegren L., 1992, A\&A, 258, 157. 